\title{
Emerging Role of Stereotactic Body Radiotherapy
}

\author{
Neeraj Jain*, Abhimanyu Rakesh and Sakshi Jain \\ Sri Guru Ram Das University of Health Sciences and Research, India
}

*Corresponding author: Neeraj Jain, Sri Guru Ram Das University of Health Sciences and Research, India.

\author{
Received Date: June 13, 2019 \\ Published Date: June 18, 2019
}

\section{Opinion}

Stereotactic Body Radiation Therapy (SBRT) also known as stereotactic ablative Radiotherapy (SABR) is a newer modality of delivering radiation therapy for many primary and secondary tumors, with good results. Multiple beams are focused to deliver high dose to single target so that tumour receive very high dose and normal tissue receive very limited dose. The aim is to deliver a high dose of radiation to a small tumour to achieve radio ablation. Patient is first immobilized and then a planning CT scan is taken. This scan is corelated with diagnostic CT, MRI and PET- CT to get the exact location and extension of tumour. Planning is then done with multiple beams focusing on tumour and with high dose per fraction aim is to achieve complete ablation. Treatment is delievered by Linacs, Cyberknife, Tomotherapy etc. Liver and lung metastases from colorectal, breast and Other cancer are most common. Studies focusing on SBRT for metastases from a single primary tumor type colorectal cancer have been published. Regardless of age, patients should have good performance status (Eastern Cooperative Oncology Group 0-1 or Karnofsky $>70$ ), with absent or stable extra hepatic disease and adequate hepatic volume and function. Number of metastasis should be less than three and size less than $6 \mathrm{~cm}$. Prescribed dose is generally very high in range of 30 to $60 \mathrm{~Gy}$ in three fractions. The toxicity profile is generally low with a G3 toxicity rate of $1-10 \%$ and the incidence of Radiation Induced Liver disease less than $1 \%$. The most common G2 toxicities included a transient hepatic enzyme levels increase over three months of SBRT and gastrointestinal, soft-tissue and bone complications, related to lesions close to the duodenum, bowel, skin and ribs. Duodenal ulceration and intestinal perforation are observed in patients with maximum doses greater than $30 \mathrm{~Gy}$ in three fractions to the duodenum and bowel. In few patients, non-traumatic rib fractures were experienced for maximum doses of 51.8 Gy and 66.2 Gy in six fractions to $0.5 \mathrm{~cm} 3$ of rib. Local control rates varied from $70 \%$ to $100 \%$ at one year and $60 \%$ to $90 \%$ at two years and correlated to lesion size $<3 \mathrm{~cm}$.

In primary lung tumour this has emerged as treatment of choice especially in peripheral tumours. In lung metastasis it is bit difficult to deliver SBRT because of movement due to respiration. Respiratory gating techniques are used, and bigger volume is treated to avoid geographical miss. Peripheral lesions are treated, and central lesions are best avoided. Common complications encountered are pneumonitis, cardiac injury, rib fracture. In solitary spinal metastasis also SBRT is being used. Single fraction high dose is given to metastatic region. In small primary tumours role of SBRT is emerging. Recent studies have suggested role in early stage Prostate cancer where a high dose in limited fractions is given and treatment Duration is reduced. Complications are early and manageable. Proctitis and cystitis are main complications but with careful planning the grade and severity can be reduced.

Stereotactic body Radiotherapy is an established modality now. Role is being investigated in Pancreatic tumours and lymph node metastasis. The modality is two-edged weapon. Wrongly planned and implemented treatment can be disastrous and life threatening. The treatment should be planned and delievered by well-trained Radiation Oncologists, Medical physicists and technologists. This requires lot of expertise patience and time to plan SBRT treatment.

\section{Acknowledgement}

None.

\section{Conflict of Interest}

No conflict of interest. 